\title{
Title: Intraneural sensory feedback restores grip force control and motor coordination while using a prosthetic hand
}

Authors: Francesco Clemente ${ }^{1 \dagger}$, Giacomo Valle ${ }^{1,2 \dagger}$, Marco Controzzi ${ }^{1}$, Ivo Strauss ${ }^{1,2}$, Francesco Iberite $^{1}$, Thomas Stieglitz ${ }^{3}$, Giuseppe Granata ${ }^{4}$, Paolo M. Rossini ${ }^{4}$, Francesco Petrini ${ }^{2}$, Silvestro Micera $^{1,2 \ddagger}$, Christian Cipriani ${ }^{1 \ddagger *}$

\author{
Affiliations: \\ ${ }^{1}$ The BioRobotics Institute, Scuola Superiore Sant'Anna, Viale R. Piaggio 34, 56025 Pontedera \\ (PI), Italy. \\ ${ }^{2}$ Bertarelli Foundation Chair in Translational Neuroengineering, Centre for Neuroprosthetics and \\ Institute of Bioengineering, School of Engineering, École Polytechnique Fédérale de Lausanne \\ (EPFL), Lausanne, Switzerland. \\ ${ }^{3}$ Laboratory for Biomedical Microtechnology, Department of Microsystems Engineering-IMTEK, \\ Bernstein Center, BrainLinks-BrainTools Cluster of Excellence, University of Freiburg, Freiburg \\ D-79110, Germany. \\ ${ }^{4}$ Institute of Neurology, Catholic University of The Sacred Heart, Policlinic A. Gemelli Foundation- \\ IRCCS, Rome, Italy. \\ *christian.cipriani@santannapisa.it \\ $\dagger,+$ Equal contribution.
}

Keywords: invasive feedback, sensory substitution, upper limb prosthetics, intraneural feedback, neural stimulation, sensorimotor control, motor learning.

\begin{abstract}
:
Objective. Tactile afferents in the human hand provide fundamental information about handenvironment interactions, which is used by the brain to adapt the motor output to the physical properties of the object being manipulated. A hand amputation disrupts both afferent and efferent pathways from/to the hand, completely invalidating the individual's motor repertoire. Although motor functions may be partially recovered by using a myoelectric prosthesis, providing functionally effective sensory feedback to users of prosthetics is a largely unsolved challenge. While past studies using invasive stimulation suggested that sensory feedback may help in handling fragile objects, none explored the underpinning, relearned, motor coordination during grasping. In this study, we aimed at showing for the first time that intraneural sensory feedback of the grip force improves the sensorimotor control of a transradial amputee controlling a myoelectric prosthesis.

Approach. We performed a longitudinal study testing a single subject (clinical trial registration number NCT02848846). A stacking cups test (CUP) performed over two weeks aimed at measuring the subject's ability to finely regulate the grip force applied with the prosthesis. A pick and lift test (PLT), performed at the end of the study, measured the level of motor coordination, and whether the subject transferred the motor skills learned in the CUP to an alien task.

Main results. The results show that intraneural sensory feedback increases the subject's ability in regulating the grip force and allows for improved performance over time. Additionally, the PLT
\end{abstract}


demonstrated that the subject was able to generalize and transfer her manipulation skills to an unknown task and to improve her motor coordination.

Significance. Our findings suggest that intraneural sensory feedback holds the potential of restoring functionally effective tactile feedback. This opens up new possibilities to improve the quality of life of amputees using a neural prosthesis.

\section{Introduction}

The tactile afferents that innervate the hand provide fundamental information about the mechanical interactions that occur between the hand and the environment, which is crucial for sensorimotor control. During object manipulation tasks, the brain uses sensory predictions and afferent signals to adapt the motor output to the physical properties of the manipulated object, as well as to monitor and update task performance [1]. In this way, humans can predict and use an adequate level of grip force required to lift an object [2], by producing highly coordinated grasping and lifting movements [3], and correcting their actions in case of unexpected events (e.g., object slip or incorrectly predicted weight) [1]. Vision provides only indirect information about the mechanical interactions of the hand with the environment, and thus people with impaired tactile sensibility exhibit difficulties with many activities of daily living [1], [2], [4], [5].

An upper limb amputation not only impairs the tactile sensibility due to the deprivation of the tactile afferents in the hand. By eradicating the physiological structures involved in the motor output (skeletal muscles, tendons and end-effector), it also invalidates the efferent pathway and the individual's motor repertoire. This aberrant situation is emphasized when the individual uses a myoelectric prosthesis, because he/she has to restore sensorimotor control using nonphysiological strategies. In particular, he/she has to re-learn how to produce new motor outputs based on a reduced amount of sensory input [6]. Indeed, while these prostheses restore an acceptable level of grasping function, they provide only indirect or substituted sensory feedback [7].

Artificial sensory feedback from a prosthetic hand can be provided in several ways, differing in stimulation principle, invasiveness and hierarchical location in the nervous system [7], [8]. Nonetheless, it is only the direct electrical stimulation of peripheral nerves or the sensory cortex, through neural electrodes, that holds the promise to restore close-to-natural and modality matched sensations [9]-[13]. Several studies proved the clinical viability of such electrodes to elicit stable sensations over long periods [11], [14], [15]; yet, only a few investigated whether neural sensory feedback conveyed salient information that is integrated into one's sensorimotor control during grasping [11], [15]. However, while these studies tackled this issue by assessing the ability of individuals to use sensory feedback about the grip force to handle fragile objects (which is relevant but limited in scope), none explored the underpinning relearned motor coordination during grasping. In fact, the latter would imply the presence of a (rebuilt) motor repertoire and would manifest in every single grasp [3], thus suggesting successful replacement of the biological hand with the prosthesis.

In this longitudinal study, we investigated whether amplitude-modulated, intraneural sensory feedback about the grip force improves grip force control and motor coordination, on a single transradial amputee while using a myoelectric prosthesis. To this aim, a stacking cups test (CUP) [16] and a pick and lift test (PLT) [17], [18] were carried out (Fig. 1). During these tests, sensory stimulation was provided through one channel of a TIME (Transverse Intrafascicular Multichannel Electrode) surgically implanted in the ulnar nerve [19]; it conveyed a continuous feedback proportional to the grip force (GF) produced by the hand prosthesis, as measured by a 
sensor in the artificial middle finger [20]. The stimulation elicited a vibratory sensation referred to the little finger of the phantom hand (see below for details), and therefore not only the feedback was not somatotopically matched but also non-physiologic. Nonetheless, as the feedback conveyed task-relevant information and was provided through the physiological channels, it was expected to be readily integrated into the individual's sensorimotor control scheme [1], [17], [21]. Results show that when the sensory feedback was active the subject did improve her manual dexterity, as measured by the CUP and PLT. To our knowledge, this is the first demonstration of a prosthetic hand with neural sensory feedback that improves grip force control and motor coordination in the user.
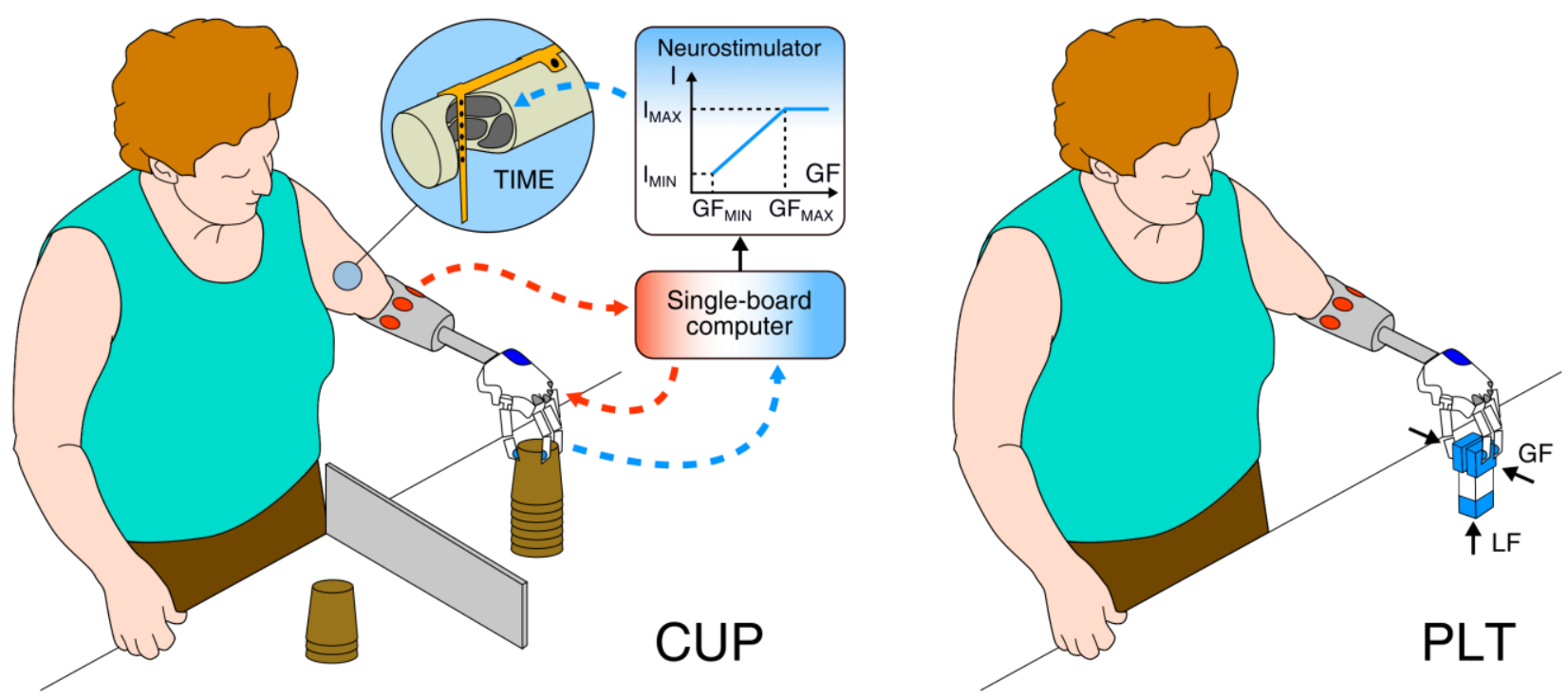

Fig. 1. Experimental setup. During the tests, the patient used a research hand prosthesis controlled through surface EMG signals, and providing neural sensory feedback through a single channel of a TIME implanted in her ulnar nerve. The amplitude of the neural stimulation was linearly mapped to the GF. The subject performed two tests: the stacking cups test (CUP) and the pick and lift test (PLT). In the former, the subject was instructed to transfer 10 plastic cups, stacked in front of her, one at a time from one stack to another as quickly as possible. As the cups were compliant, the subject had to regulate the grip force in order to pick single cups. In the PLT, the subject was instructed to repeatedly grip, lift, replace and release a test-object capable of measuring the grip forces (GF) and load forces (LF). A trial was considered successful if the subject was able to perform it while avoiding that the GF exceeded a fixed virtual "breaking" threshold.

\section{Methods}

\section{Subject and implant}

The ethical approval of this study was obtained by the Institutional Ethics Committee of the Policlinic A. Gemelli Foundation-IRCCS at the Catholic University, in Rome, Italy, where the surgery was performed and by the Italian Ministry of Health. Informed consent was signed. All experiments were conducted in accordance with relevant guidelines and regulations (clinical trial registration number NCT02848846). 
The subject was a 53 years old female, who had undergone transradial (proximal third of the forearm) amputation in December 2015 following a work accident. The subject took part in a long-term study (six months) of intraneural electrodes and as such in July 2017, was exposed through a $15 \mathrm{~cm}$-long skin incision in the left arm performed in general anesthesia - to implant four TIMEs [19] in an optimized version with embedded adhesion promotion layers for improved longevity: two in the median nerve (one proximal, one distal) and two in the ulnar nerve (one proximal, one distal) [22]. The tests described in this manuscript were performed four months after implantation and took place in six experimental sessions across four weeks (Fig. 2B).

During the surgery, the median nerve proved to have a cross-sectional area of $4-5 \mathrm{~mm}^{2}$, i.e. much smaller than average [23]. This, together with the fact that the electrical stimulation of this nerve never evoked sensations in the phantom hand throughout the long term study, suggested that most of the sensory fibres innervating the hand prior to the amputation atrophied (see [15] for a more detailed description of this issue). Hence, in this work, one channel of the TIME inserted proximally in the ulnar nerve was used for stimulation (see below).
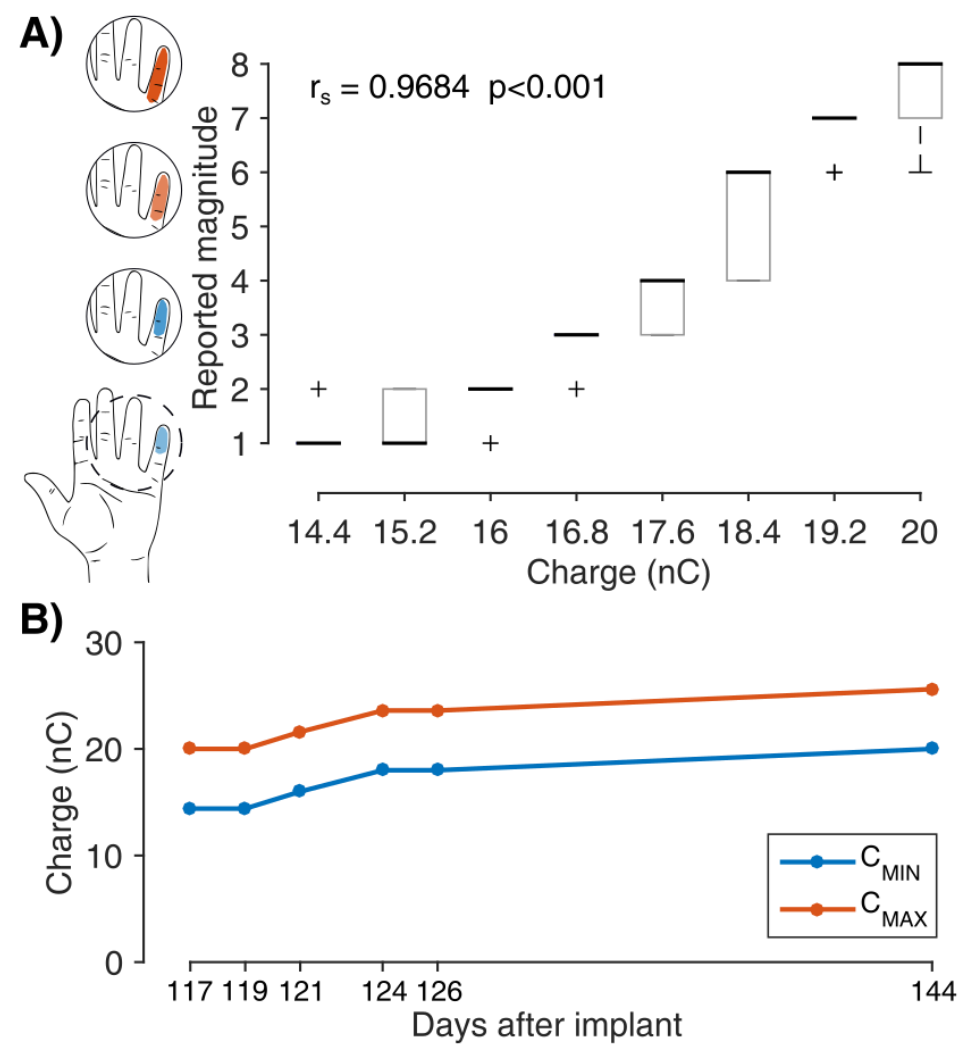

Fig. 2. Neural stimulation characterization. A) Perceived magnitude and areas of referred sensations as a function of the injected charge in the channel of the TIME used in the tests. The perceived sensation was always described as a vibration. The thick black lines indicate the median $(n=10)$, the grey boxes denote the $25^{\text {th }}$ and $75^{\text {th }}$ percentiles, the whiskers represent the minimum and maximum values and the crosses represent outliers. The injected charge and the perceived magnitude were highly correlated $\left(r_{s}=0.9684\right.$, $\mathrm{p}<0.001)$. B) Minimum charge needed to evoke a sensation $\left(\mathrm{C}_{\mathrm{MIN}}\right)$ and the maximum one that produced an intense but not painful sensation $\left(\mathrm{C}_{\mathrm{MAX}}\right)$, as a function of the test day. Note that the CUP was performed on days 117-126, whereas the PLT was performed on day 144 . 


\section{Prosthetic hand}

The subject was fitted with a research prosthesis comprising (i) an anthropomorphic robotic hand with grip force sensors (IH2 Azzurra hand, Prensilia SRL, Italy), (ii) a recording and stimulation device (Neural Interface Processor, Ripple LLC, US) that acquired the EMG signals from the surface electrodes and conveyed sensory feedback about the grip force to the subject through one channel of the TIME inserted proximally in the ulnar nerve, and (iii) a single-board computer (Raspberry Pi 3, Raspberry Pi Foundation, UK) running a custom multithreaded C application that interpreted the subject's muscle contractions to open and close the hand, and controlled the neural stimulator based on the sensors readout (Fig. 1). The thumb of the robot hand was physically constrained to the palm in order to allow stable tripod grasps between the thumb, the index and the middle fingers.

Four surface EMG signals were acquired from the residual muscles in the forearm. In particular, two electrodes picked up signals from the dorsal and two from the ventral side of the forearm. The signals were sampled at $2 \mathrm{kHz}$ and digitally filtered using a $4^{\text {th }}$ order band-pass $(15-375 \mathrm{~Hz})$ Butterworth IIR filter, and a notch filter to remove the $50 \mathrm{~Hz}$ power line interference. A robust three-state (open, close, rest) k-Nearest Neighbor $(\mathrm{kNN}, \mathrm{k}=3)$ classifier with a decision-based velocity ramp [24] was used to control the hand. The classifier was fed with the waveform length of each EMG channel, computed over a window of $100 \mathrm{~ms}$, and allowed a proportional control of the speed of the robotic hand movements. The classifier was retrained every time the EMG electrodes were newly placed (i.e. every experimental day). The simplicity of the setup was a guarantee of consistent performance across the days and thus of experimental repeatability.

The neural stimulator delivered biphasic, charge-balanced, cathodic-first, rectangular pulses at a frequency of $50 \mathrm{~Hz}$. The pulse-width was fixed $(80 \mu \mathrm{s})$, while the amplitude was linearly mapped to the grip force (Fig. 1) recorded by a miniaturized load cell [25] in the middle finger and updated in real time (20 ms output rate), akin to [20]. In particular, the injected charge varied between $\mathrm{C}_{\mathrm{MIN}}$ and $\mathrm{C}_{\mathrm{MAX}}$, which corresponded to the contact threshold of the hand $(0.2 \mathrm{~N})$ and a GF of $4.4 \mathrm{~N}$, respectively. The latter was empirically chosen and corresponded to the breaking threshold of the PLT (see below). $\mathrm{C}_{\text {MIN }}$ and $\mathrm{C}_{\mathrm{MAX}}$ were experimentally assessed before each session, and corresponded to the just perceivable sensation ( 1 in a scale 1-10- $\mathrm{C}_{\mathrm{MIN}}$ ) and to the charge that produced a comfortable but not painful one (8 in a scale 1-10- $\mathrm{C}_{\mathrm{MAX}}$ ) (Fig. 2B). Besides $C_{\text {MIN }}$ and $\mathrm{C}_{\text {MAX }}$ the type and location of the perceived sensation was logged before each session, and the perceived magnitude of stimulation-evoked sensations was measured using a free magnitude estimation paradigm [9], [10], [26]. To this aim, the pulse amplitude was modulated (between $180 \mu \mathrm{A}$ and $250 \mu \mathrm{A}$ in steps of $10 \mu \mathrm{A})$, keeping the pulse-width fixed (80 $\mu$ s) (i.e., 14.4 $\mathrm{nC}-20 \mathrm{nC}$ in steps of $0.8 \mathrm{nC}$ ) (Fig. 2A).

\section{Stacking cups test (CUP)}

The CUP required the subject to transfer one of 10 plastic cups at a time (beer cups: $500 \mathrm{ml}$, polypropylene, $4.54 \mathrm{~g}$, base diameter $6 \mathrm{~cm}$, apex diameter $10 \mathrm{~cm}$, and height $12 \mathrm{~cm}$ ) from one stack to another, as quickly as possible (Fig. 1 and Supplementary Movie S1). The test demanded fine motor control of grasping, because lifting a single cup from the stack was possible only by producing GF below $2.2 \mathrm{~N}$ (experimentally measured). A larger GF caused multiple cups to be lifted and transferred at the same time. When this occurred, an error was counted and a single cup was counted as transferred. The performance metrics of the CUP were the percentage of singularly transferred cups $(\mathrm{P})$ and the time $(\mathrm{T})$ required to complete the test. 


\section{Pick and lift test (PLT)}

The PLT is a well-established procedure in motor control studies [18], used to assess the integration of sensorimotor control paradigms. In the PLT, the subject, standing in front of a table, is instructed to repeatedly grip, lift, replace and release at a self-selected speed, a test-object capable of measuring the GF and load forces (LF). Specifically, in this study the task consisted in (i) moving the arm to reach the object $(40 \times 45 \times 130 \mathrm{~mm} ; \sim 200 \mathrm{~g})$ with the prosthesis, (ii) grasping the object, (iii) lifting the object a few centimeters above the table, (iv) putting the object back on the table, and, finally, (v) releasing the object (Fig. 1). A trial was considered to be successful if the subject was able to perform it while avoiding that the GF exceeded a fixed "breaking" threshold (experimentally set to $4.4 \mathrm{~N}$ after pilot experiments). The minimum GF required to lift the test-object with the robot hand without slippage was $\sim 2.2 \mathrm{~N}$. Conversely, if the subject generated a GF larger than the threshold, the test-object virtually broke; this event was signaled to the subject through an acoustic signal. The GF and LF recorded by the test-object were both acquired and used to assess the motor coordination offline.

\section{Experimental protocol}

The CUP was repeated across 12 sessions, once with (F-ON) and once without (F-OFF) neural sensory feedback. The order of the conditions was randomized and balanced between sessions. The 12 sessions were spread over five days in a period of two weeks (days 117, 119, 121, 124, 126 after implant, Fig. 2) in order to evaluate mid-term learning effects. The PLT was repeated six times, 18 days after the last session of the CUP (day 144 after implant, Fig. 2). Each repetition included one series of 20 trials with F-OFF or F-ON, with the conditions randomized across repetitions (F-OFF, F-OFF, F-ON, F-ON, F-OFF, F-ON). Thus, in addition to motor coordination, the PLT measured whether the subject transferred the motor skills learned in the CUP to an alien task [27].

By demanding fine GF control (CUP) and motor coordination (PLT), together the CUP and the PLT provided two independent measures of integration of the intraneural sensory feedback in the sensorimotor control of the subject.

\section{Data analysis and statistical methods}

In the CUP, the percentage of individually transferred cups was compared between F-ON and FOFF conditions using the Wilcoxon rank-sum test. The same was performed to compare the time needed to complete the task. Spearman's rank correlation coefficients $\left(\mathrm{r}_{\mathrm{s}}\right)$ were computed to evaluate monotonic trends in transferred cups and completion time across sessions in order to assess learning effects.

In the PLT, the GF-LF coordination was quantified through the temporal delay between the instants when the GF and LF reached 50\% of the LF at lift-off [3], [17]. This was compared between the F-ON and F-OFF conditions using a Wilcoxon rank-sum test.

In all cases, a $\mathrm{p}$ value $<0.05$ was considered statistically significant.

\section{Results}


The intraneural stimulation elicited a stable sensation of vibration referred to the little finger for the duration of this study, as assessed before each experimental session (Fig. 2A). However, the amount of charge that elicited a just perceivable sensation, $\mathrm{C}_{\mathrm{MIN}}$, and the one that produced a comfortable but not painful sensation, $\mathrm{C}_{\mathrm{MAX}}$, slightly increased with time, ranging from 14.4 to 20 $\mathrm{nC}$ and from 34.4 to $40 \mathrm{nC}$, respectively (Fig. 2B). $\mathrm{C}_{\mathrm{MIN}}$, and $\mathrm{C}_{\mathrm{MAX}}$ corresponded to the contact threshold of the hand and a $4.4 \mathrm{~N} \mathrm{GF}$, respectively, as measured by the GF sensor in the hand. Moreover, the area of the perceived sensation increased with increasing charge albeit it never extended beyond the palmar region of the little finger (Fig. 2A). The stability of the elicited sensations, as verified by the characterization, allowed to run this longitudinal study under consistent experimental conditions.

During the CUP, the subject grasped stronger in the F-OFF condition than in the F-ON condition, as revealed by the GF time series (Fig. 3A). In other words, when available, the subject took advantage of the intraneural sensory feedback to limit the GF applied on the cups to avoid grasping more than one at a time. Therefore, in all sessions (12 out of 12), the subject performed better in the F-ON than in the F-OFF condition, in terms of both percentage of singularly transferred cups $\left(\mathrm{P}_{\mathrm{F}-\mathrm{ON}}=81.7 \pm 5.8 \%\right.$ vs $\mathrm{P}_{\mathrm{F}-\mathrm{OFF}}=54.2 \pm 9.9 \%$, Wilcoxon rank-sum test, $\mathrm{p}<0.001$, Fig. $3 \mathrm{~B})$ and time needed to complete the task $\left(\mathrm{T}_{\mathrm{F}-\mathrm{ON}}=67.2 \pm 10.7 \mathrm{~s} \mathrm{~T}_{\mathrm{F}-\mathrm{OFF}}=105.9 \pm 20.4 \mathrm{~s}\right.$, Wilcoxon rank-sum test, p<0.001, Fig. 3C).
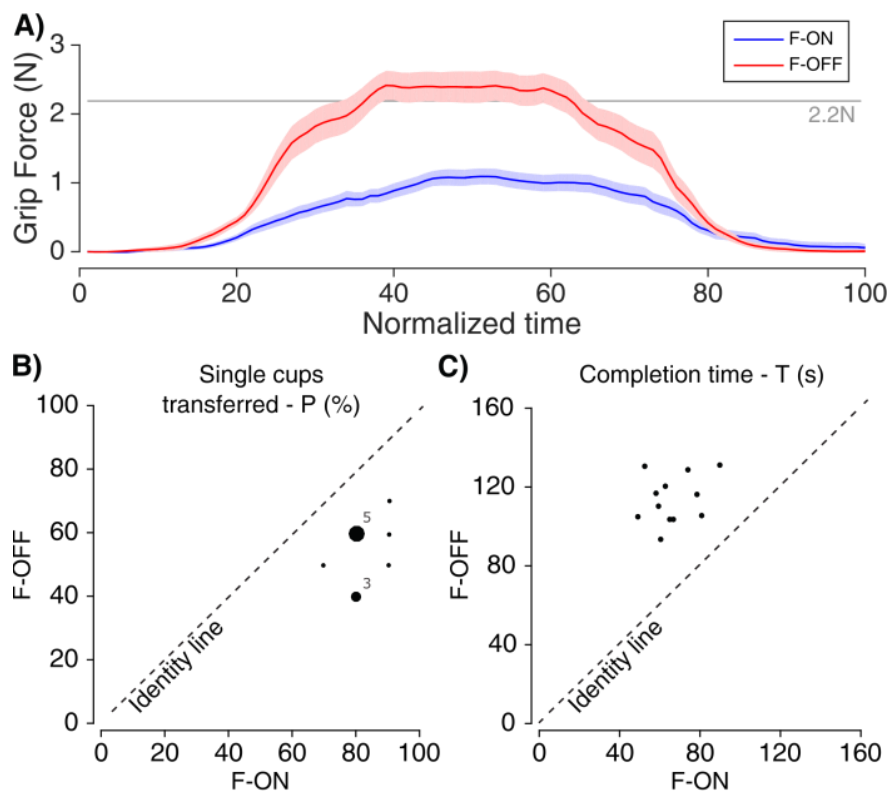

Fig. 3. Results from the stacking cups test (CUP). A) Grip force profiles of single trials, showing that the subject was able to regulate (limit) the grip force applied on the cups when intraneural sensory feedback was available (F-ON condition). Thick lines represent the mean, shaded areas represent the standard error of the mean $(n=30)$. B) Scatter plot comparing the percentage of cups transferred singularly (performance $\mathrm{P}$ ) in the two conditions. In all sessions, less cups were transferred singularly in F-OFF than in F-ON condition (Wilcoxon rank-sum test, $\mathrm{p}<0.001$ ). C) Scatter plot comparing the completion time to transfer 10 cups in the two conditions. In all sessions, more time was required in the F-OFF than in the F-ON condition to complete the task (Wilcoxon rank-sum test, $\mathrm{p}<0.001$ ). Each marker represents a single experimental session. The size of the markers is proportional to the number of sessions with the same outcome. 
The percentage of single cups transferred over time (Fig. 4) did not change significantly (Spearman's rank correlation $\mathrm{r}_{\mathrm{s}}=0.28, \mathrm{p}=0.36$ and $\mathrm{r}_{\mathrm{s}}=0.02, \mathrm{p}=0.95$ for $\mathrm{F}-\mathrm{ON}$ and F-OFF, respectively), although a more pronounced positive trend was found in the F-ON condition (Fig. 4A). More interestingly, the time to complete the test demonstrated a significant negative trend $\left(\mathrm{r}_{\mathrm{s}}=-0.58, \mathrm{p}=0.04\right)$ in the F-ON condition, which instead was not observed in the F-OFF condition $\left(r_{s}=0.08, p=0.79\right)($ Fig. 4B).

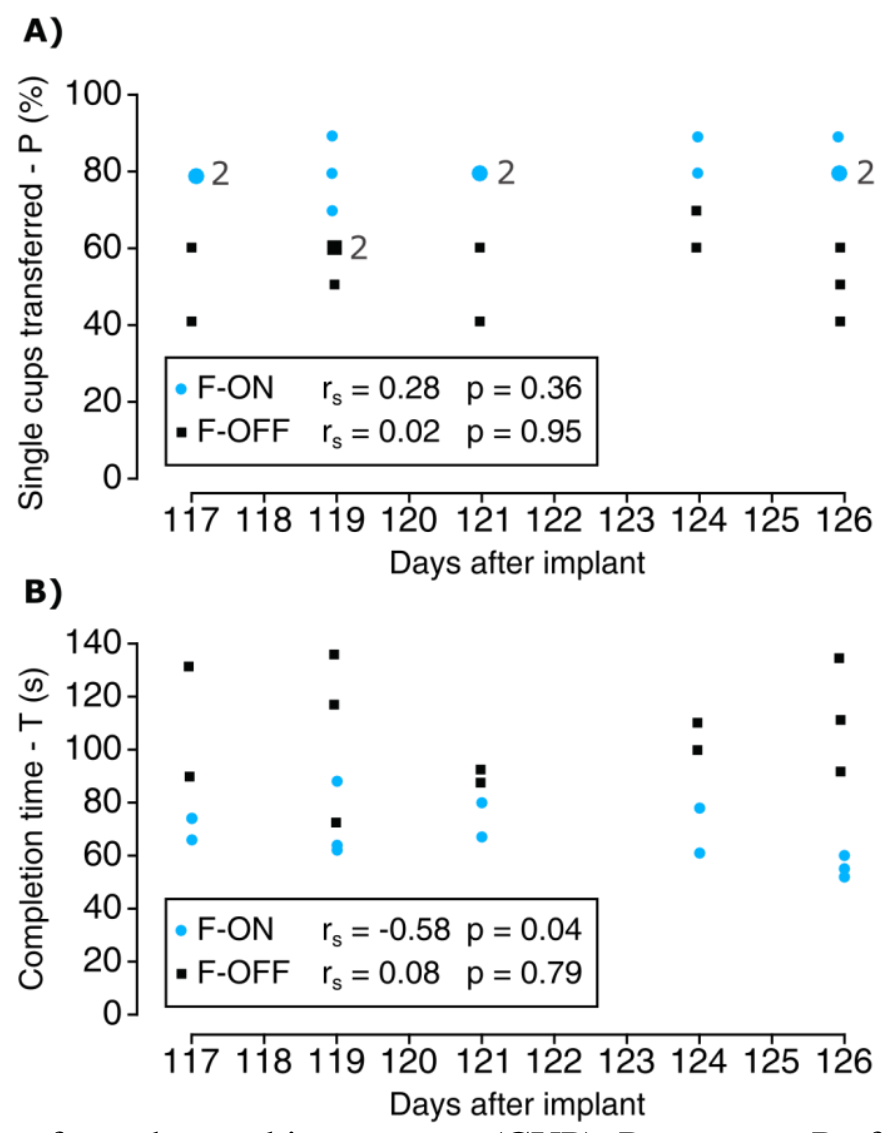

Fig. 4. Results over time from the stacking cup test (CUP). Percentage $P$ of cups transferred singularly (A) and time $T$ required by the subject to complete the test (B) for each experimental session as a function of test day. $\mathrm{T}$ in the $\mathrm{F}-\mathrm{ON}$ condition showed a significant negative trend over time $\left(\mathrm{r}_{\mathrm{s}}=-0.58, \mathrm{p}=0.04\right)$, while no significant change was found in the F-OFF condition. The size of the markers is proportional to the number of sessions with the same outcome. Blue circles and black squares indicate the F-ON and FOFF condition, respectively.

In the PLT, the subject showed better motor coordination in the F-ON condition (Fig. 5A), as demonstrated by the higher temporal correlation between the GF and LF time series [28]. This was confirmed by the statistically shorter temporal delay between the GF and LF onsets in the FON condition (211:180 ms, median:IQR) with respect to F-OFF (320:239 ms) (Wilcoxon ranksum test, $\mathrm{p}<0.001$, Fig. 5B). Namely, the median delay was reduced by $34 \%$ when the neural feedback was available. A faster behavior was observed also in the load phase duration, i.e. the time from the first contact with the object to lift-off. It proved faster in the F-ON (330:238 ms) than in the F-OFF condition (480:297 ms) (Wilcoxon rank-sum test, $\mathrm{p}<0.001$ ).

Collectively, the statistically better grip force control, learning trends, motor coordination profiles (associated to a transfer of motor skills) and confidence/speed in performing the manipulative 
tasks, proved that the neural feedback restored a sophisticated sensorimotor control of the prosthetic hand in the individual.

A)
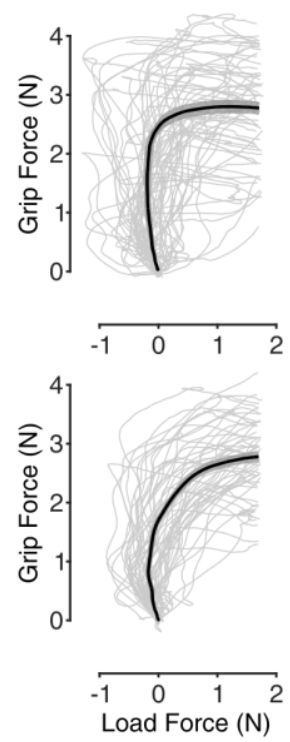

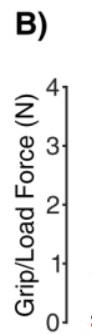

Synced at
thumb contact

Grip force F-OFF
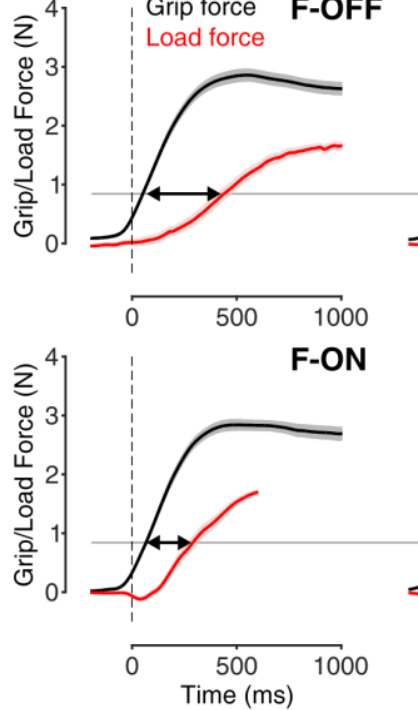
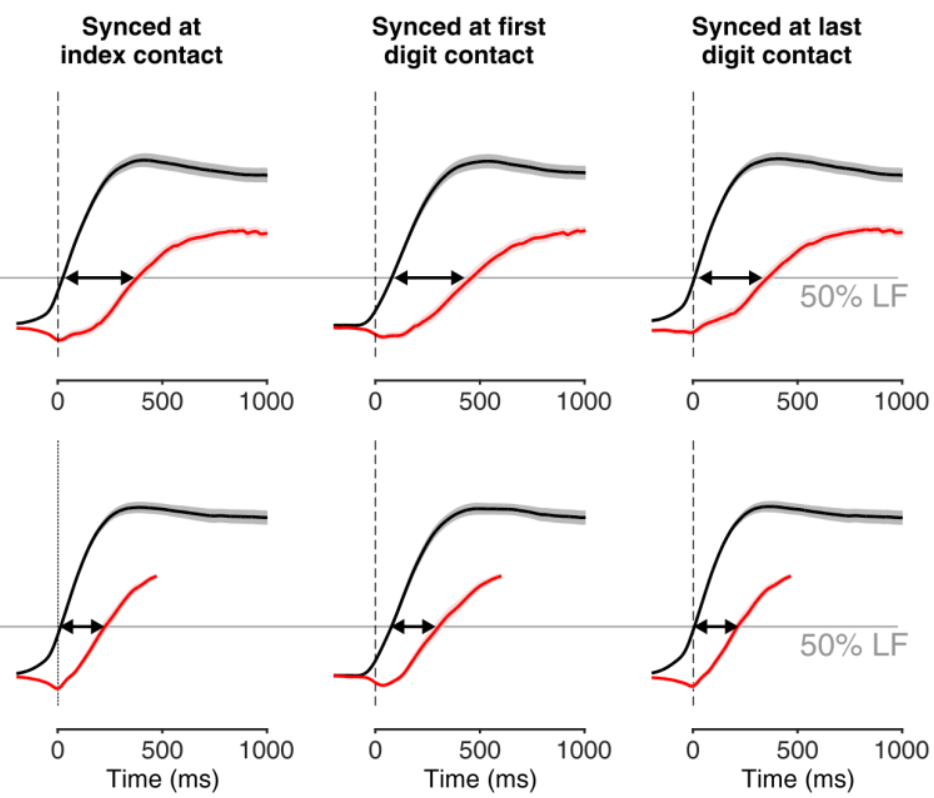

Fig. 5. Motor coordination during the PLT. The subject exhibited better motor coordination during the PLT in the F-ON condition. This is reflected in the higher temporal correlation between the GF and LF (A) and confirmed by the smaller temporal delay between the rising of the LF with respect to the GF (B). The difference between the two conditions was statistically significant (Wilcoxon rank-sum test, $\mathrm{p}<0.001$ ). Solid lines represent means and filled areas represent standard error of the mean $(n=60)$.

\section{Discussion}

This is the first time that neural sensory feedback showed to systematically improve grip force control and motor coordination in functional grasping tasks without the need to occlude vision (as, in example, in [12], [13], [20]). The closest study is the work by Tan and colleagues [11] where the ability of a single subject in pulling stems from cherries was evaluated. With this clever albeit original test, they found that epineural sensory feedback improved the subject's ability in not crushing the cherries, i.e. in regulating the GF applied on them. By using a more deterministic/rigorous approach and two independent tests, here we support but also considerably extend their findings by showing that the neural feedback allows for learning and improves motor coordination.

Specifically, akin to humans with anesthetized fingertips [2], [3], the subject showed poor temporal correlation between the GF and the LF when only vision was available (Fig. 5). Conversely, when the neural feedback about GF was turned on, the patterns demonstrated closer to a mature grasp behavior [1], [3], corroborating the thesis that, albeit non-somatotopically matched nor entirely natural, this feedback allowed to build an internal model of the motor task, and because of this to grasp in a predictive feedforward fashion [1]. This argument is supported by the duration of the load phase observed during F-ON (330 ms median), remarkably close to that found in healthy humans (i.e. $300 \mathrm{~ms}$ ) while grasping similar weights [1], [3]. The duration in 
the F-OFF condition, 50\% longer than F-ON (480 ms median), suggests, instead, that the subject exploited a purely feedback mechanism based on the incoming visual information.

The outcomes of the CUP matched and complemented those of the PLT. The neural feedback of the GF effectively boosted the performance. When enabled, the subject transferred $\sim 25 \%$ more cups (akin to previous studies [11], [29]), completed the task $35 \%$ faster and with time learned to go even faster (Fig. 4). The lack of a learning effect and the statistically lower performance in the F-OFF condition are striking indicators that -when available- the sensory feedback was integrated in the control scheme and used to refine the internal model of the motor task. Visual feedback alone demonstrated ineffective to bring about similar motor skills in the timeframe of this study.

In support of our thesis, it is worth noting that the subject was trained to use the sensory feedback during certain activities (i.e. the CUP) and was then able to generalize and transfer this skill to an alien task, such as the PLT. Previous studies on this matter, where subjects were trained and tested with the same task [11], [12], [30] demonstrated the learning process (acquisition) only; however, it is the ability to apply what has been learned to new task variants (transfer) that entails true learning skills [27].

Although it would be desirable to use the neural interface to restore biomimetic sensations [9][13], in the current implant all sites in the two ulnar nerve electrodes elicited vibration sensations, from largely overlapping regions of the phantom little finger or wrist [15]. Hence, it was not possible to map the sensors of two or more digits involved (i.e. thumb, index and middle fingers) to multiple sites of the electrodes, and only one site (one channel) was chosen for stimulation. This circumstance turned out to unveil an interesting feature of the neural feedback, not investigated earlier. Namely, our results were achieved with a sensory feedback that was neither somatotopically (sensation in the little instead of the thumb, index and middle fingers) nor modality matched (vibration instead of pressure). These outcomes were not obvious as reinterpreting sensory information is known to tax the cognitive systems [31]. Even in the case of hand transplants, where re-sutured nerves significantly re-innervate biological sensors — which should be theoretically superior to any artificial sensory feedback - functional (sensory) recovery is unsatisfactory [32], [33] unless the patient is in the early teens or younger [34], or if neural plasticity is favored [35]. Successful reinterpretation of time-continuous sensory feedback yielding improved motor control was demonstrated only in a few cases where modality-matched stimulation was provided. In particular, when re-innervated mechanoreceptors after targeted sensory reinnervation [36] were targeted by haptic stimulators [30] or when natural tactile perceptions were elicited by peripheral nerve cuff electrodes [11]. We hypothesize that sensory reinterpretation was made possible in our setup because targeting the nerve fascicles elicited an ample range of neural codes (cf. Fig 2), akin to those involved in natural grasping or in somatotopic and modality matched haptic stimulation [30], [37] where stimuli are mediated by biological sensors. We advocate that sensory reinterpretation also arose from the 'Bayesian logic' of all perception, i.e., the brain's remarkable ability to detect statistical correlations in sensory inputs in constructing useful perceptual representations of the world [38]. In fact, the artificial tactile inflow conveyed salient information correlated/coupled with visual and accompanying acoustic sensory inputs. Arguably, and in agreement with other perceptual illusions [39], this bottom-up process proved resistant to top-down knowledge of body reactions to stimuli and because of this may contribute to promote self-attribution of the prosthesis [40]. Finally, although not explicitly assessed, adaptation to continuous electrical stimulation of the nerve proved not an issue. As suggested by a recent study by Graczyk and colleagues [41], the brain diminishes the 
response to constant and therefore uninformative stimulation and becomes more responsive to changes which are more informative [42].

While the positive results described earlier suggest that sensory feedback may allow to grasp in a predictive fashion (i.e. that the artificial feedback allows them to re-build a motor repertoire which includes the hand prosthesis) [11], [29], [30], this was never verified or quantified so far. An asset of this study, that allowed to infer on how the subject grasped, is that we used more sophisticated assessment tools like the PLT. The latter is a well-established method borrowed from the neuroscience literature, to assess motor coordination during grasp [18] and to compare e.g., healthy vs. impaired participants, unimpaired vs. anesthetized digits, etc. [3], [17]. We invite researchers in this area to adopt these tools to gain novel insights into the assessment of advanced artificial limbs. From a clinical perspective, the confirmation that sensory feedback helps building an internal model of the grasp holds the promise to reduce the abandonment ratio of myoelectric prostheses due to excessive cognitive burden [43]. Interestingly, this achievement could be possible even with a single, clinically viable, stimulation channel [14]. This vision supports the use of neural stimulation in a much more convincing way than earlier because restoring the ability to grasp in a natural way is clearly the first priority for a hand replacement.

Finally, this work was limited in some respects and thus invites a number of interesting studies on sensorimotor control of artificial hands with neural sensory feedback. While it is clear that our subject improved grip force control and motor coordination when provided with neural feedback from a single stimulation channel in the ulnar nerve, it still remains to be assessed whether this can be generalized to a larger clinical population, to multiple electrode channels and target nerves. In addition, the PLT is a relatively simple task, requiring arm-hand coordination only. Whether these outcomes may extend to more complex tasks, involving the coordination of multiple body movements, requires further investigations. Moreover, although a reliable assessment of functional activities was allowed in the laboratory, the subject could not bring the prosthesis away for continuous use due to technical and ethical constraints. In fact, she could use the prosthesis only three days a week. In addition, a simple charge modulation strategy was used to convey feedback. Whether more natural feedback, somatotopic and/or continuous use may entail even more natural control remains an intriguing yet open question.

Acknowledgments: The authors are deeply grateful to the patient who participated in this study. The authors would also like to thank Dr. Vito Monaco for suggestions on the statistical analysis. Funding: This work was funded by the European commission under the NEBIAS project (FP7 FET, GA \# 611687). Competing interests: C.C., M.C. and F.C. hold shares in Prensilia Srl, a company that manufactures robotic hands as the one used in this work. F.M.P. and S.M. hold shares of Sensars Neuroprosthetics Sarl, a start-up company dealing with potential commercialization of neurocontrolled artificial limbs. Data and materials availability: Data available on request.

\section{Supplementary Materials:}

Movie S1. Stacking cups test (CUP). The subject performs the test in the F-ON experimental condition. The performance is displayed in real time on the top right corner of the video. At the end of the video, the experimenter asks the subject to transfer as many cups as possible to demonstrate her ability of generating large grip forces. The test was performed on day 126 after implantation. 


\section{References and Notes:}

[1] R. S. Johansson and J. R. Flanagan, "Coding and use of tactile signals from the fingertips in object manipulation tasks," Nat. Rev. Neurosci., vol. 10, no. 5, pp. 345-359, 2009.

[2] A. G. Witney, A. Wing, J.-L. Thonnard, and A. M. Smith, "The cutaneous contribution to adaptive precision grip," Trends Neurosci., vol. 27, no. 10, pp. 637-643, 2004.

[3] J. Monzée, Y. Lamarre, and A. M. Smith, "The effects of digital anesthesia on force control using a precision grip," J. Neurophysiol., vol. 89, no. 2, pp. 672-683, 2003.

[4] D. A. Nowak and J. Hermsdörfer, "Grip force behavior during object manipulation in neurological disorders: toward an objective evaluation of manual performance deficits," Mov. Disord., vol. 20, no. 1, pp. 11-25, 2005.

[5] J.-L. Thonnard, C. Detrembleur, and P. Y. K. Van Den Bergh, "Assessment of hand function in a patient with chronic sensory demyelinating neuropathy," Neurology, vol. 49, no. 1, pp. 253-257, 1997.

[6] D. S. Childress, "Closed-loop control in prosthetic systems: historical perspective," Ann. Biomed. Eng., vol. 8, no. 4-6, pp. 293-303, 1980.

[7] C. Antfolk, M. D’Alonzo, B. Rosén, G. Lundborg, F. Sebelius, and C. Cipriani, "Sensory feedback in upper limb prosthetics," Expert Rev. Med. Devices, vol. 10, no. 1, pp. 45-54, 2013.

[8] C. Pasluosta, P. Kiele, and T. Stieglitz, "Paradigms for restoration of somatosensory feedback via stimulation of the peripheral nervous system," Clin. Neurophysiol., vol. 129, no. 4, pp. 851-862, 2018.

[9] S. N. Flesher et al., "Intracortical microstimulation of human somatosensory cortex," Sci. Transl. Med., vol. 8, no. 361, 2016.

[10] E. L. Graczyk, M. A. Schiefer, H. P. Saal, B. P. Delhaye, S. J. Bensmaia, and D. J. Tyler, "The neural basis of perceived intensity in natural and artificial touch," Sci. Transl. Med., vol. 8, no. 362, p. 362ra142-362ra142, 2016.

[11] D. W. Tan, M. A. Schiefer, M. W. Keith, J. R. Anderson, J. Tyler, and D. J. Tyler, “A neural interface provides long-term stable natural touch perception," Sci. Transl. Med., vol. 6, no. 257, p. 257ra138-257ra138, Oct. 2014.

[12] S. Wendelken et al., "Restoration of motor control and proprioceptive and cutaneous sensation in humans with prior upper-limb amputation via multiple Utah Slanted Electrode Arrays (USEAs) implanted in residual peripheral arm nerves," J. Neuroeng. Rehabil., vol. 14, no. 1, pp. 1-17, 2017.

[13] K. Horch, S. Meek, T. G. Taylor, and D. T. Hutchinson, "Object discrimination with an artificial hand using electrical stimulation of peripheral tactile and proprioceptive pathways with intrafascicular electrodes," IEEE Trans. Neural Syst. Rehabil. Eng., vol. 19, no. 5, pp. 483-489, 2011.

[14] M. J. Ortiz-Catalan, B. Håkansson, and R. Brånemark, “An osseointegrated humanmachine gateway for long-term sensory feedback and motor control of artificial limbs," Sci. Transl. Med., vol. 6, no. 257, p. 257re6-257re6, 2014.

[15] F. M. Petrini et al., "Six-months assessment of a hand prosthesis with intraneural tactile feedback," Submitted, 2018. 
[16] M. Markovic et al., "The clinical relevance of advanced artificial feedback in the control of a multi-functional myoelectric prosthesis," J. Neuroeng. Rehabil., vol. 15, no. 1, 2018.

[17] C. Cipriani, J. L. Segil, F. Clemente, R. F. ff. Weir, and B. B. Edin, "Humans can integrate feedback of discrete events in their sensorimotor control of a robotic hand," Exp. Brain Res., vol. 232, no. 11, pp. 3421-3429, 2014.

[18] R. S. Johansson and G. Westling, "Coordinated isometric muscle commands adequately and erroneously programmed for the weight during lifting task with precision grip," Exp. Brain Res., vol. 71, no. 1, pp. 59-71, 1988.

[19] T. Boretius et al., "A transverse intrafascicular multichannel electrode (TIME) to interface with the peripheral nerve," Biosens. Bioelectron., vol. 26, no. 1, pp. 62-69, 2010.

[20] S. Raspopovic et al., "Restoring Natural Sensory Feedback in Real-Time Bidirectional Hand Prostheses," Sci. Transl. Med., vol. 6, no. 222, p. 222ra19-222ra19, 2014.

[21] D. A. Nowak, S. Glasauer, and J. Hermsdörfer, "How predictive is grip force control in the complete absence of somatosensory feedback?," Brain, vol. 127, no. 1, pp. 182-192, 2004.

[22] W. Schady, S. Braune, S. Watson, H. E. Torebjörk, and R. Schmidt, "Responsiveness of the somatosensory system after nerve injury and amputation in the human hand," Ann. Neurol., vol. 36, no. 1, pp. 68-75, 1994.

[23] I. Delgado-Martinez, J. Badia, A. Pascual-Font, A. Rodriguez-Baeza, and X. Navarro, "Fascicular topography of the human median nerve for neuroprosthetic surgery," Front. Neurosci., vol. 10, no. JUL, pp. 1-13, 2016.

[24] A. M. Simon, L. J. Hargrove, B. A. Lock, and T. A. Kuiken, "A decision-based velocity ramp for minimizing the effect of misclassifications during real-time pattern recognition control," IEEE Trans. Biomed. Eng., vol. 58, no. 8, pp. 2360-2368, 2011.

[25] C. Cipriani et al., "Closed-loop controller for a bio-inspired multi-fingered underactuated prosthesis," in Proceedings of the IEEE International Conference on Robotics and Automation, 2006, vol. 2006, pp. 2111-2116.

[26] S. S. Stevens, "The direct estimation of sensory magnitudes: loudness," Am. J. Psychol., vol. 69, no. 1, pp. 1-25, 1956.

[27] R. D. Seidler and D. C. Noll, "Neuroanatomical Correlates of Motor Acquisition and Motor Transfer," J. Neurophysiol., vol. 99, no. 4, pp. 1836-1845, 2008.

[28] H. Forssberg, A. C. Eliasson, H. Kinoshita, R. S. Johansson, and G. Westling, "Development of human precision grip I: Basic coordination of force," Exp. Brain Res., vol. 85, no. 2, pp. 451-457, 1991.

[29] F. Clemente, M. D’Alonzo, M. Controzzi, B. B. Edin, and C. Cipriani, "Non-invasive, temporally discrete feedback of object contact and release improves grasp control of closed-loop myoelectric transradial prostheses," IEEE Trans. Neural Syst. Rehabil. Eng., vol. 24, no. 12, pp. 1314-1322, 2016.

[30] K. Kim and J. E. Colgate, "Haptic feedback enhances grip force control of sEMGcontrolled prosthetic hands in targeted reinnervation amputees," IEEE Trans. Neural Syst. Rehabil. Eng., vol. 20, no. 6, pp. 798-805, 2012.

[31] B. Rosén, G. Lundborg, L. B. Dahlin, J. Holmberg, and B. Karlson, "Nerve repair: correlation of restitution of functional sensibility with specific cognitive capacities," $J$. Hand Surg. Am., vol. 19, no. 4, pp. 452-458, 1994. 
[32] J. T. Shores, G. Brandacher, and W. P. A. Lee, "Hand and upper extremity transplantation: An update of outcomes in the worldwide experience," Plast. Reconstr. Surg., vol. 135, no. 2, p. 351e-360e, 2015.

[33] C. L. Kaufman, B. Blair, E. Murphy, and W. B. Breidenbach, “A new option for amputees: transplantation of the hand," J. Rehabil. Res. Dev., vol. 46, no. 3, pp. 395-404, 2009.

[34] G. Lundborg and B. Rosén, "Sensory relearning after nerve repair," Lancet, vol. 358, no. 9284, pp. 809-810, 2001.

[35] C. M. Galtrey, R. A. Asher, F. Nothias, and J. W. Fawcett, "Promoting plasticity in the spinal cord with chondroitinase improves functional recovery after peripheral nerve repair," Brain, vol. 130, no. 4, pp. 926-939, 2007.

[36] T. A. Kuiken, P. D. Marasco, B. A. Lock, R. N. Harden, and J. P. A. Dewald, "Redirection of cutaneous sensation from the hand to the chest skin of human amputees with targeted reinnervation," Proc. Natl. Acad. Sci. U. S. A., vol. 104, no. 50, pp. 20061-20066, 2007.

[37] D. Leonardis, M. Solazzi, I. Bortone, and A. Frisoli, “A 3-RSR Haptic Wearable Device for Rendering Fingertip Contact Forces," IEEE Trans. Haptics, vol. 10, no. 3, pp. 305-316, 2017.

[38] K. C. Armel and V. S. Ramachandran, "Projecting sensations to external objects: evidence from skin conductance response," Proc. R. Soc. B Biol. Sci., vol. 270, no. 1523, pp. 14991506, 2003.

[39] M. Botvinick and J. Cohen, "Rubber hands 'feel' touch that eyes see," Nature, vol. 391, no. 6669, p. 756, 1998.

[40] M. D’Alonzo, F. Clemente, and C. Cipriani, "Vibrotactile stimulation promotes embodiment of an Alien hand in amputees with phantom sensations," IEEE Trans. Neural Syst. Rehabil. Eng., vol. 23, no. 3, pp. 450-457, 2015.

[41] E. L. Graczyk, B. Delhaye, M. A. Schiefer, S. J. Bensmaia, and D. J. Tyler, "Sensory adaptation to electrical stimulation of the somatosensory nerves," J. Neural Eng., vol. 15, no. 4, p. 046002, 2018.

[42] N. Brenner, W. Bialek, and R. de Ruyter van Steveninck, "Adaptive rescaling maximizes information transmission,” Neuron, vol. 26, no. 3, pp. 695-702, 2000.

[43] E. A. Biddiss, D. Beaton, and T. T. Chau, "Consumer design priorities for upper limb prosthetics," Disabil. Rehabil. Assist. Technol., vol. 2, no. 6, pp. 346-357, 2007. 